\title{
Análisis de las acciones de juego en pádel masculino profesional
}

\author{
Analysis of game actions in professional male padel \\ Óscar Mellado-Arbelo ${ }^{1}$, Ernest Baiget Vidal ${ }^{2}$, Marc Vivès Usón ${ }^{3}$ \\ 1 Sports Sciences Research Group, Institut Nacional d’Educació Física de Catalunya, Universitat de Barcelona, España. \\ 2 Sport Performance Analysis Research Group, Universitat de Vic-Universitat Central de Catalunya, España. \\ 3 Grup de Recerca Consolidat, Institut Nacional d'Educació Física de Catalunya, Universitat de Barcelona, España.
}

\author{
CORRESPONDENCIA: \\ Óscar Mellado Arbelo \\ oscar.mellado.arbelo@gmail.com
}

CÓMO CITAR EL ARTÍCULO:

Mellado-Arbelo, O., Baiget, E., \& Vivès, M. (2019). Análisis de las acciones de juego en pádel masculino profesional. Cultura Ciencia_Deporte, 14(42), 191-201.

Recepción: enero 2017 • Aceptación: julio 2017

\section{Resumen}

Los objetivos de este estudio fueron: (a) definir una herramienta estandarizada de análisis técnico-táctico en pádel, (b) realizar un análisis descriptivo de las acciones de juego y aspectos tácticos en el pádel profesional masculino. Se utilizó un diseño observacional construyendo un instrumento de observación específico diseñado ad hoc. Se analizaron 8 partidos del circuito profesional World Padel Tour 2014 y un total de 8.541 acciones de golpeo, registrando para cada una el jugador que realiza el golpe, su ubicación en la pista, el tipo de golpe, la trayectoria, profundidad y recorrido de la bola y la consecuencia del golpe. Entre los tipos de golpe destacan las voleas de revés y derecha (16.6\% y $13 \%$ respectivamente), revés (12.4\%), primer servicio (11.2\%) y derecha (10.7\%). Predominan las trayectorias cruzadas $(57.5 \%)$ sobre las paralelas $(42.5 \%)$. La zona desde la que más se golpea es la de fondo (49.1\%), seguida de la zona de red (26.4\%) y la de transición (19.3\%). Se registraron porcentajes del $87.6 \%$ en golpes interceptados, $5.5 \%$ de errores no forzados, $4.7 \%$ de puntos ganadores y $2.1 \%$ de errores forzados. Los golpes con los que se registró un mayor número de puntos ganadores fueron el remate $(45.9 \%)$, bajada de pared de derecha $(7.9 \%)$, voleas de derecha y revés $(5.7 \%$ y $3.4 \%$ respectivamente) y bandeja (3.5\%). En conclusión, los golpes más frecuentes en un partido de pádel profesional son la derecha y revés de fondo, voleas, bandeja y remate. En cada punto suelen sucederse largos intercambios de golpes, sobre todo cruzados, que mayoritariamente finalizan con errores no forzados del rival o puntos ganadores de volea, remate y bandeja.

Palabras clave: Análisis táctico, Metodología observacional, Alto rendimiento deportivo.

\section{Abstract}

The goals of this study were (a) to define a technical and tactical analysis standardized tool for padel, (b) carry a descriptive analysis of play actions and tactical aspects in masculine professional padel. An observational design was employed, constructing an observational instrument designed ad hoc. A total of eight padel matches (8.541 actions) pertaining to World padel Tour 2014 professional circuit were analyzed. Data was registered regarding the player executing each shot, his situation in the playing court, the type of shot, the trajectory, depth and route of the ball and the consequence of the analyzed shot for a total of 8,581 shot actions. Amongst the types of shots executed the following stand out: forehand and backhand volleys (16.6\% and $13 \%$ respectively), backhands ( $12.4 \%)$, first serve (11.2\%) and forehand (10.7\%). Crossed trajectories (57.5\%) prevail over parallel trajectories $(42.5 \%)$. The zone in the playing field from which the most shots were executed was the back court (49.1\%), followed by the net zone (26.4\%) and the transition zone (19.3\%). Percentages were registered for intercepted shots (87.6\%), unforced errors $(5.5 \%)$, winning points $(4.7 \%)$ and forced errors $(2.1 \%)$. The shots with which the greatest number of winning points were registered were the smash (45.9\%), forehand drop shot $(7.9 \%)$, the forehand and backhand volleys $(5.7 \%$ and $3.4 \%$ respectively) and the tray shot (3.5\%). In conclusion, the most frequent shots in a professional padel match are drives and backhands from the back court, volleys, tray shots and smashes. In every game point there often is a long exchange of shots, specially crossed shots, which mainly finish with unforced errors by the opponent or volley, smash or tray shot winning points.

Key words: Tactical analysis, Observational methodology, High performance sport. 


\section{Introducción}

El pádel se constituye como un deporte de reciente creación (Sánchez-Alcaraz, 2013), relativamente joven en el ámbito del alto rendimiento deportivo, con una importancia creciente en los últimos años (Ruiz \& Lorenzo, 2008). En la última década se ha producido un gran incremento del número de licencias en España, pasando de 13.698 en el año 2005 a 56.085 licencias en 2015 (Courel-Ibáñez, Sánchez-Alcaraz, García \& Echegaray, 2017; Federación Española de Pádel, 2015).

En el ámbito competitivo se celebran varias pruebas a lo largo del año, tanto a nivel autonómico como nacional e internacional. En categoría absoluta destacan las competiciones organizadas por la Federación Española de Pádel (FEP) (Campeonato de España por equipos), los torneos celebrados por las federaciones autonómicas puntuables para la clasificación nacional en categorías oro, plata y bronce y, por último, las pruebas organizadas por el World Pádel Tour (WPT). El WPT es un circuito de ámbito internacional, con clasificación propia que consta de 18 pruebas anuales categorizadas en diferentes tipos de pruebas como son los Challenger, Open, Máster y Masters Finals. La principal diferencia entre ellas son las condiciones de acceso en función de la clasificación de los jugadores, siendo las pruebas Challenger las de menor categoría y el Máster Final la de mayor.

El pádel, del mismo modo que otros deportes de raqueta, forma parte de los denominados deportes abiertos en los que es necesaria una constante toma de decisiones (O’Donoghue \& Ingram, 2001), por lo que los aspectos relacionados con la táctica y las destrezas cognitivas se erigen fundamentales (GonzálezCarvajal, 2003; Courel, Cañas, Sánchez-Alcaraz \& Alarcón-Guerrero, 2014). En este sentido, el análisis de las acciones técnico-tácticas que se dan durante el juego puede aportar un valioso conocimiento aplicable a la planificación del entrenamiento y al desarrollo de estrategias competitivas (Courel, Sánchez-Alcaraz \& Cañas, 2015). Durante los últimos años se ha incrementado el conocimiento sobre los elementos técnicotácticos en los deportes de raqueta, concretamente en tenis se han analizado las características de los golpeos (Hughes \& Clarke, 1995; Over \& O’Donoghue, 2008), las distancias recorridas (Costa et al., 2016; Yuzo, Tardelli, Roveri, Shoiti, Machado \& Arruda, 2016; Reid \& Duffield, 2014), los aspectos temporales del juego (Fernández, Méndez-Villanueva \& Pluim, 2006; Kilit, Şenel, Arslan \& Can, 2016) o el perfil de los puntos jugados (Jhonson \& McHugh, 2006). Asimismo, se han analizado las características estructurales del juego y de la competición en otros deportes de raqueta como el bádminton (Cabello \& González, 2003; Hong \& Tong, 2000) o el squash (Girard, Chevalier, Habard, Scibens, Hot \& Millet, 2007; Sanchis, González, López, Dorado \& Chaberren, 1998).

Debido a la reciente creación del pádel, que data de finales de la década de los años sesenta (Navarro, 2014), todavía son escasos los estudios que determinen la exigencia competitiva de este deporte y las acciones específicas de juego. Algunos de los contenidos técnicotácticos estudiados actualmente son la frecuencia de ejecución de diferentes tipos de golpes (derecha, revés, globo, volea y remate) en jugadores de nivel nacional (Sánchez-Alcaraz, 2014a; Sañudo, De Hoyo \& Carrasco, 2008) y profesional (Priego et al., 2013). También han sido estudiados los tipos de golpes en función de las zonas de la pista desde la que se ejecutan (Almonacid, 2012), la precisión de los golpeos en función del nivel de los jugadores (Courel, Cañas \& Sánchez-Alcaraz 2016) o la influencia de las variables situacionales en la eficacia y duración de los puntos en jugadores profesionales (Courel-Ibáñez \& Sánchez-Alcaraz, 2017; Ramón-Llin, 2013). Asimismo, las cuestiones de uso y eficacia de diferentes tipos de golpeo para provocar situaciones tácticas favorables han sido abordadas en varias publicaciones (Muñoz-Marín et al., 2017). En última instancia encontramos en la bibliografía científica varios estudios centrados en la influencia del género en la estructura temporal y las acciones de juego del pádel profesional (García-Benítez, Pérez-Bilbao, Echegaray \& Felipe, 2016; Torres-Luque, Ramírez, Cabello-Manrique, Nikolaidis \& Alvero-Cruz, 2015; Sánchez-Alcaraz, 2014b), el uso y la eficacia de diferentes tipos de golpeo para provocar situaciones tácticas favorables (MuñozMarín et al., 2017). Sin embargo, no existen hasta la fecha investigaciones centradas específicamente en el análisis de los golpeos, sino que estas han sido analizadas de forma complementaria en trabajos que definen las características fisiológicas de los jugadores y estructurales de la competición (Carrasco, Romero, Sañudo \& De Hoyo, 2011; Priego et al., 2013). Las clasificaciones utilizadas en estudios previos (Sañudo et al., 2008; Priego et al., 2013; Almonacid, 2012) no consideran algunos parámetros inherentes a la propia acción de golpeo, como son el jugador que golpea la bola, zona de golpeo, tipo específico de golpe, trayectoria de la bola, profundidad de la misma, recorrido y resultado del golpeo.

El principal objetivo de este estudio es diseñar el primer instrumento de observación computerizado de análisis de acciones de juego en pádel que aporte un sistema de categorías propio y específico de este deporte. Asimismo, se plantea como segundo objetivo el análisis de las acciones de juego en pádel masculino profesional. 


\section{Método}

\section{Diseño}

El diseño observacional utilizado es nomotético, dada la pluralidad de los participantes, puntual y multidimensional, ya que los parámetros a analizar se clasifican en diversos criterios y categorías. Se trata de un proceso no participante porque el observador no interactúa con los sujetos durante la misma. Se analizaron un total de 8 partidos correspondientes a las fases finales (cuartos de final, semifinales y finales) de diferentes pruebas del circuito WPT 2014 en categoría masculina.

En esta competición el acceso queda restringido a los treinta primeros jugadores de la clasificación de la FEP y los 160 primeros de la clasificación propia de la WPT, confeccionándose un cuadro final de 28 parejas (56 jugadores).

\section{Participantes}

Se observaron 20 jugadores profesionales, con una edad media de $34.5 \pm 5.7$ años una experiencia mínima de 3 años compitiendo como profesionales y una media de $138.2 \pm 6.8$ partidos acumulados en torneos del circuito WPT.

Al tratarse de un estudio observacional en entorno natural (competición oficial) y de difusión pública no fue necesario el consentimiento informado de los deportistas.

\section{Instrumento observacional}

El instrumento de observación: Sistema de Observación en Pádel (SOPD) (Tabla 1), diseñado ad hoc, se fundamenta en las publicaciones científicas precedentes sobre análisis del juego en tenis y pádel (Gorospe et al., 2005; Brody, 2006; Corrales Sañudo et al., 2008; Priego et al., 2013; Almonacid, 2012; Fernández de Ossó \& León-Prados, en prensa), libros técnicos (González-Carvajal, 2003; Moncaut, 2003; Moyano, 2011; Navarro, 2014; González-Carvajal, 2003; Courel et al., 2014) y el manual de formación de técnicos de la FEP. Consta de once categorías, que recogen datos del jugador que realiza cada golpeo a lo largo del punto, su ubicación en la pista (Figura 1), el tipo de golpeo que realiza, la trayectoria que sigue la bola tras ser golpeada, la profundidad del bote o de golpeo por parte del oponente, su recorrido y el resultado del golpe analizado. Asimismo, en cada punto se registró el tanteo del juego que se disputaba, el jugador que estaba al servicio, el marcador y el set.

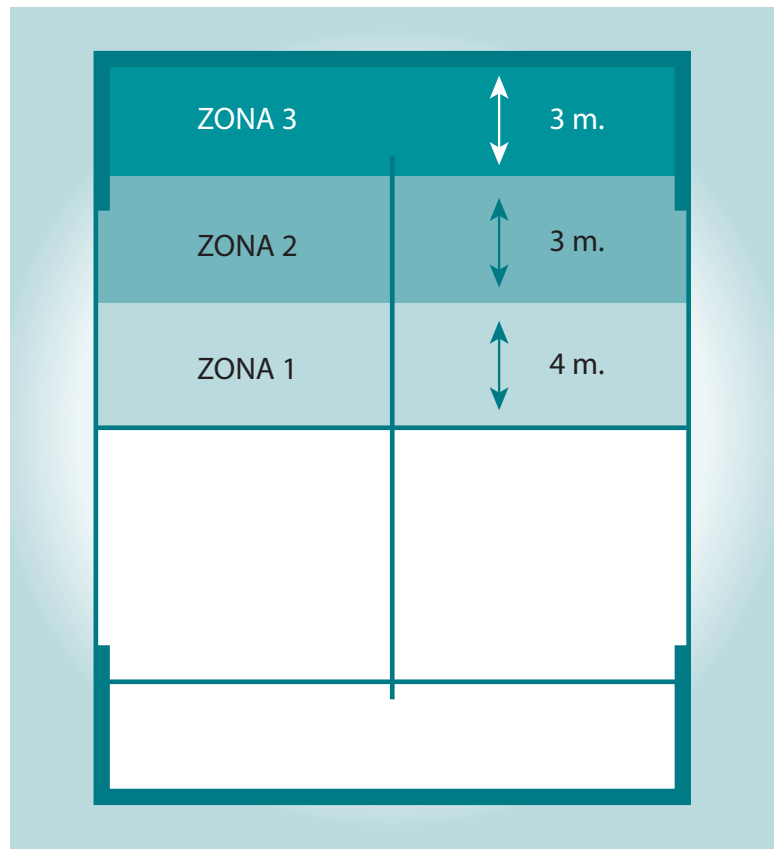

Figura 1. Zonas de la pista.

\section{Procedimiento}

Se tomaron como material de análisis los vídeos filmados por la propia organización del WPT. Todos ellos se obtuvieron a través de la plataforma web oficial del circuito, donde pueden ser descargados de forma libre en formato MPEG-4 con una calidad de 1920x1080 píxeles (Full HD-1080p). Se trata de filmaciones efectuadas con una sola cámara, fija, situada en uno de los fondos de la pista, registrando un plano general picado de la pista. Todo el tratamiento de edición y calibración de vídeos previos a la observación y registro de datos se realizó con el software Adobe Premiere CS5. Para definir las zonas de juego se trazaron unas líneas paralelas superpuestas a la pista en el vídeo original. Se informatizó el proceso observacional a través del software LINCE v.1.2.1. (Gabín, Camerino, Anguera \& Castañer, 2012), diseñando un instrumento de observación a medida con criterios y categorías específicas para el presente estudio (Tabla 1).

\section{Control de la calidad del dato}

La calidad del dato se determinó a través de procesos de validez y fiabilidad. La validez de constructo se garantizó mediante la consistencia conceptual, extraída en el marco conceptual del pádel y citado en el apartado de "instrumento observacional" y mediante un panel de expertos compuesto por 8 especialistas de pádel (titulados por la FEP). Dichos especialistas obtuvieron un acuerdo del $100 \%$ para cada una de las categorías. La validación 
Tabla 1. Instrumento observacional SOPD.

\begin{tabular}{|c|c|c|}
\hline Criterio & Código & Descripción \\
\hline \multirow{4}{*}{ JUGADOR QUE GOLPEA } & J1 & Jugador de la posición del drive de la pareja ganadora \\
\hline & $\mathrm{J} 2$ & Jugador de la posición del revés de la pareja ganadora \\
\hline & $\mathrm{J} 3$ & Jugador de la posición del drive de la pareja perdedora \\
\hline & J4 & Jugador de la posición del revés de la pareja perdedora \\
\hline \multirow[t]{7}{*}{ UBICACIÓN DEL JUGADOR } & $\mathrm{Z1}$ & Zona 1 (Desde la red hasta 4 metros) \\
\hline & $\mathrm{Z2}$ & Zona 2 (Desde la línea de servicio hasta 4 metros de la red) \\
\hline & $\mathrm{Z3}$ & Zona 3 (Desde la pared de fondo hasta la línea de servicio, 3 metros) \\
\hline & $\mathrm{Z1C}$ & Zona 1 en posición cambiada \\
\hline & $\mathrm{Z2C}$ & Zona 2 en posición cambiada \\
\hline & $\mathrm{Z3C}$ & Zona 3 en posición cambiada \\
\hline & $\mathrm{FP}$ & Fuera de pista \\
\hline \multirow[t]{29}{*}{ TIPO DE GOLPE } & SQ1 & Primer servicio \\
\hline & SQ2 & Segundo servicio \\
\hline & $\mathrm{D}$ & Derecha \\
\hline & $\mathrm{R}$ & Revés \\
\hline & SPD & Salida de pared de derecha \\
\hline & SPR & Salida de pared de revés \\
\hline & PLD & Pared lateral de derecha \\
\hline & PLR & Pared lateral de revés \\
\hline & $\mathrm{RD}$ & Reja de derecha \\
\hline & $\mathrm{RR}$ & Reja de revés \\
\hline & DPAD & Doble pared que abre de derecha \\
\hline & DPAR & Doble pared que abre de revés \\
\hline & DPCD & Doble pared que cierra de derecha \\
\hline & DPCR & Doble pared que cierra de revés \\
\hline & DPAGD & Doble pared que abre con giro de derecha \\
\hline & DPAGR & Doble pared que abre con giro de revés \\
\hline & DPCGD & Doble pared que cierra con giro de derecha \\
\hline & DPCGR & Doble pared que cierra con giro de revés \\
\hline & CPFD & Contra-pared de fondo de derecha \\
\hline & CPFR & Contra-pared de fondo de revés \\
\hline & CPLD & Contra pared lateral de derecha \\
\hline & CPLR & Contra pared lateral de revés \\
\hline & BPD & Bajada de pared de derecha \\
\hline & BPR & Bajada de pared de revés \\
\hline & VD & Volea de derecha \\
\hline & VR & Volea de revés \\
\hline & $\mathrm{BD}$ & Bandeja \\
\hline & FR & Finta de remate \\
\hline & $\mathrm{RM}$ & Remate \\
\hline \multirow[t]{4}{*}{ TRAYECTORIA DE LA BOLA } & C & Cruzado (La bola bota o es interceptada en el lado de la pista opuesto diagonalmente al que es golpeada) \\
\hline & $\mathrm{P}$ & Paralelo (La bola bota o es interceptada en el mismo lado de la pista en el que es golpeada) \\
\hline & FDPXA & Fuera de pista hacia dentro por arriba \\
\hline & FDPXP & Fuera de pista hacia dentro por puerta \\
\hline \multirow[t]{3}{*}{ PROFUNDIDAD DE LA BOLA } & $\mathrm{Z1}$ & Zona 1 \\
\hline & $\mathrm{Z2}$ & Zona 2 \\
\hline & $\mathrm{Z3}$ & Zona 3 \\
\hline \multirow[t]{18}{*}{ RECORRIDO DE LA BOLA } & NB & No bota \\
\hline & $1 \mathrm{~B}$ & 1 bote \\
\hline & BPFX3 & Bota, golpea la pared de fondo, sale de la pista por 3 metros \\
\hline & BXF & Bota, sale de la pista por el fondo \\
\hline & BFV & Bota, golpea la pared de fondo y vuelve a la pista de jugador que golpea \\
\hline & BPFRJ & Bota, pared de fondo, reja \\
\hline & BPL & Bota, golpea pared lateral \\
\hline & BPLPF & Bota, golpea pared lateral, golpea pared de fondo \\
\hline & BRJ & Bota, golpea la reja \\
\hline & $\mathrm{BX} 3$ & Bota, sale de la pista por 3 metros \\
\hline & BXP & Bota, sale de la pista por la puerta \\
\hline & RD & Golpea directamente la red \\
\hline & $\mathrm{PF}$ & Golpea directamente la pared de fondo \\
\hline & $\mathrm{PL}$ & Golpea directamente la pared lateral \\
\hline & RJ & Golpea directamente la reja \\
\hline & FPI & Sale directamente fuera de la pista \\
\hline & GJ & Golpea al jugador oponente \\
\hline & BGJ & Bota, golpea al jugador oponente \\
\hline \multirow[t]{4}{*}{ RESULTADO DEL GOLPE } & PG & Punto ganador \\
\hline & $\mathrm{EF}$ & Error forzado \\
\hline & ENF & Error no forzado \\
\hline & $\mathrm{Gl}$ & Golpe interceptado \\
\hline TANTEO & $\mathrm{X}-\mathrm{X}$ & Puntuación del partido $(15,30,40$, AV, Juego). \\
\hline \multirow{4}{*}{ JUGADOR AL SERVICIO } & J1 & Jugador Posición drive de la pareja ganadora \\
\hline & $\mathrm{J} 2$ & Jugador Posición revés de la pareja ganadora \\
\hline & $\mathrm{J} 3$ & Jugador posición drive de la pareja perdedora \\
\hline & J4 & Jugador posición revés de la pareja perdedora \\
\hline SET & $\mathrm{x}$ & 1, 2 ó 3. (Todos los partidos se juegan al mejor de 3 sets) \\
\hline MARCADOR & $\mathrm{X}-\mathrm{X}$ & Donde $\mathrm{X}$ toma valores de 0 a 7 \\
\hline
\end{tabular}


Tabla 2. Tipología de los golpes.

\begin{tabular}{|c|c|c|c|c|}
\hline Familia de golpe & Tipo de golpe & $\mathrm{N}$ & $\%$ & $M \pm D E$ \\
\hline \multirow{5}{*}{ Golpes Directos } & Volea de revés & 1428 & 16.6 & $178.5 \pm 43.4$ \\
\hline & Volea de derecha & 1115 & 13.0 & $139.4 \pm 38.1$ \\
\hline & Bandeja & 514 & 6.0 & $64.3 \pm 31.7$ \\
\hline & Remate & 512 & 6.0 & $64.0 \pm 12.2$ \\
\hline & Finta de remate & 76 & 0.9 & $9.5 \pm 5.1$ \\
\hline \multirow{4}{*}{ Golpes Indirectos } & Revés & 1060 & 12.4 & $132.5 \pm 38.6$ \\
\hline & Primer servicio & 965 & 11.2 & $120.6 \pm 25.3$ \\
\hline & Derecha & 914 & 10.7 & $114.3 \pm 25.6$ \\
\hline & Segundo servicio & 97 & 1.1 & $12.1 \pm 2.7$ \\
\hline \multirow{8}{*}{ Golpes Indirectos con Pared Simple } & Salida de pared de derecha & 534 & 6.2 & $66.8 \pm 11.1$ \\
\hline & Salida de pared de revés & 347 & 4.0 & $43.4 \pm 15.8$ \\
\hline & Bajada de pared de derecha & 189 & 2.2 & $23.7 \pm 6.7$ \\
\hline & Pared lateral de revés & 155 & 1.8 & $19.4 \pm 9.4$ \\
\hline & Reja de revés & 86 & 1.0 & $10.8 \pm 4.5$ \\
\hline & Pared lateral de derecha & 74 & 0.9 & $9.3 \pm 8.2$ \\
\hline & Bajada de pared de revés & 33 & 0.4 & $4.1 \pm 1.3$ \\
\hline & Reja de derecha & 24 & 0.3 & $3.0 \pm 2.4$ \\
\hline \multirow{8}{*}{ Golpes Indirectos con Doble Pared } & Doble pared que abre con giro de derecha & 83 & 1.0 & $10.4 \pm 6.2$ \\
\hline & Doble pared que cierra de revés & 75 & 0.9 & $9.4 \pm 3.2$ \\
\hline & Doble pared que abre de revés & 56 & 0.7 & $7.0 \pm 2.3$ \\
\hline & Doble pared que abre de derecha & 35 & 0.4 & $4.4 \pm 3.0$ \\
\hline & Doble pared que cierra de derecha & 23 & 0.3 & $9.4 \pm 3.2$ \\
\hline & Doble pared que abre con giro de revés & 15 & 0.2 & $1.9 \pm 1.5$ \\
\hline & Doble pared que cierra con giro de revés & 15 & 0.2 & $1.9 \pm 1.8$ \\
\hline & Doble pared que cierra con giro de derecha & 2 & 0.1 & $0.3 \pm 0.4$ \\
\hline \multirow{5}{*}{ Golpes Indirectos Contra-Pared } & Contra-pared fondo de derecha & 129 & 1.5 & $16.1 \pm 6.1$ \\
\hline & Contra-pared fondo de revés & 13 & 0.2 & $1.6 \pm 2.4$ \\
\hline & Contra pared lateral de revés & 8 & 0.1 & $1 \pm 1.2$ \\
\hline & Contra-pared lateral de derecha & 4 & 0.1 & $0.5 \pm 0.5$ \\
\hline & Total & 8581 & 100 & \\
\hline
\end{tabular}

se efectuó mediante un cuestionario virtual (formulario Google) en el que los expertos debían manifestar si estaban de acuerdo o no con la categorización de cada criterio. La fiabilidad en la observación se determinó mediante la concordancia interobservador en 200 acciones de juego. Se obtuvo un valor de 0.94 en el coeficiente Kappa (Cohen, 1968) en el contraste de los observadores que participaron en el estudio. El grado de concordancia intraobservador arrojó coeficiente Kappa (Cohen, 1968) de 0.97 en el análisis de las 200 acciones de juego.

\section{Análisis estadístico}

Se registraron un total de 8.581 golpeos para la totalidad de los partidos analizados. El registro de los datos realizado en el software LINCE fue exportado en formato .CSV al paquete estadístico SPSS 22.0 (IBM, Chicago, IL, USA), con el que se realizó todo el procesamiento y análisis de datos. El análisis descriptivo muestra las distintas categorías del estudio a través de su frecuencia y porcentajes totales y media y desviación estándar $(\mathrm{M} \pm \mathrm{DE})$ por partido. El análisis de normali- dad de las variables fue realizado mediante los estadísticos de Kolmogorov-Smirnov y de Shapiro-Wilk. Las diferencias entre tipo de los golpes, trayectoria, resultado y zonas de golpeo fueron determinadas mediante un análisis de la varianza (ANOVA) con la corrección de Bonferroni en las variables con distribución normal y la prueba de Kruskal-Wallis y Mann-Whitney para muestras independientes en el caso de las variables con distribución no normal. El nivel de significación fue establecido en $p<0.05$ para todas las variables.

\section{Resultados}

Tipología de los golpes

En la tabla 2 se detallan las frecuencias absolutas y relativas totales y los promedios por partido de los diferentes tipos de golpeo. Destacan, por orden de frecuencia de aparición, la volea de revés (16.6\%), la volea de derecha (13.0\%), el revés (12.4\%), el primer servicio $(11.2 \%)$ y la derecha (10.7\%). En golpes directos, 
existen diferencias significativas entre todos ellos ( $\mathrm{p}<$ $0.05)$ excepto entre la bandeja y el remate $(p=1.000)$. En golpes indirectos no existen diferencias significativas entre los golpes de derecha, revés y primer servicio ( $p$ > 0.05). Sin embargo, encontramos diferencias significativas entre el segundo servicio y el resto de los golpes indirectos $(\mathrm{p}<0.001)$. En los golpes indirectos con pared simple existen diferencias entre salida de pared de derecha y todos los golpes $(\mathrm{p}<0.05)$ excepto salida de pared de revés $(p=0.109)$. Existen diferencias entre salida pared de revés y todos los golpes ( $\mathrm{p}$ $<0.05)$ excepto salida pared de derecha $(\mathrm{p}=0.109)$, bajada pared de derecha $(\mathrm{p}=0.429)$ y pared lateral de revés $(p=0.069)$. No existen diferencias entre pared lateral de revés, reja de revés, pared lateral de derecha, bajada de pared de revés y reja de revés ( $p$ > 0.05). En cuanto a golpes indirectos con doble pared, no existen diferencias significativas entre doble pared que cierra de derecha, doble pared que cierra de revés y doble pared que abre de revés $(\mathrm{p}>0.05)$ y si existen diferencias entre estos 3 golpes y el resto de los golpes con doble pared ( $\mathrm{p}<0.05)$. Tampoco existen diferencias entre doble pared que abre con giro de revés, doble pared que cierra con giro de revés y doble pared que cierra con giro de derecha $(\mathrm{p}>0.05)$ y si existen diferencias entre estos tres golpes y el resto $(p<0.05)$. Por otro lado, existen diferencias entre doble pared que abre de derecha y doble pared que abre con giro de derecha y doble pared que cierra de revés $(p<0.05)$. Entre los golpes de contra-pared, se han hallado diferencias significativas entre contra-pared fondo de derecha y el resto de los golpes $(p<0.001)$. No existen diferencias entre contra-pared fondo revés, contra-pared lateral de revés y contra-pared lateral de derecha ( $\mathrm{p}>0.05)$.

\section{Trayectoria de los golpes}

La tabla 3 muestra los datos obtenidos en el análisis de las trayectorias de los golpeos.

Se observa un predominio de las trayectorias cruzadas (57.5\%) sobre las paralelas (42.5\%), existiendo diferencias significativas entre los 4 tipos de trayectoria de los golpes $(p<0.05)$, excepto entre los golpes de fuera-dentro de pista por puerta y fuera-dentro de pista por alto $(\mathrm{p}=1.000)$. Asimismo, el número de bolas con trayectoria fuera-dentro de pista, que se corresponde con las pelotas recuperadas desde fuera de la pista, resulta prácticamente insignificante.

\section{Resultado de los golpes}

La tabla 4 muestra los resultados obtenidos en cuanto a la consecución de los golpeos. Existe un marcado
Tabla 3. Trayectoria de los golpes.

\begin{tabular}{lccc}
\hline Trayectoria de la bola & $\mathbf{n}$ & $\%$ & $\mathbf{M} \pm \mathbf{D E}$ \\
\hline Cruzado & 4912 & 57.2 & $614.0 \pm 144.6$ \\
Paralelo & 3631 & 42.3 & $453.9 \pm 105.7$ \\
Fuera-dentro de pista por puerta & 22 & 0.3 & $2.8 \pm 1.4$ \\
Fuera-dentro de pista por alto & 16 & 0.2 & $2.0 \pm 1.7$ \\
Total & $\mathbf{8 5 8 1}$ & $\mathbf{1 0 0}$ & \\
\hline
\end{tabular}

Tabla 4. Resultado del golpe.

\begin{tabular}{lccc}
\hline Resultado del golpe & $\mathrm{n}$ & $\%$ & $\mathrm{M} \pm \mathrm{DE}$ \\
\hline Golpe interceptado & 7521 & 87.6 & $940.1 \pm 207.3$ \\
Error no forzado & 476 & 5.5 & $59.5 \pm 17.8$ \\
Punto ganador & 407 & 4.7 & $50.9 \pm 10.4$ \\
Error forzado & 177 & 2.1 & $22.13 \pm 5.0$ \\
Total & $\mathbf{8 5 8 1}$ & $\mathbf{1 0 0}$ & \\
\hline
\end{tabular}

Tabla 5. Zonas de golpeo.

\begin{tabular}{lccc}
\hline Zonas de golpeo & $\mathbf{n}$ & $\%$ & $\mathbf{M} \pm \mathrm{DE}$ \\
\hline Zona 3 & 4217 & 49.1 & $527.3 \pm 116.7$ \\
Zona 1 & 2264 & 26.4 & $282.9 \pm 66.1$ \\
Zona 2 & 1652 & 19.3 & $206.5 \pm 54.4$ \\
Zona 3 cruzado & 155 & 1.8 & $19.4 \pm 9.9$ \\
Zona 2 cruzado & 143 & 1.7 & $17.8 \pm 12.8$ \\
Zona 1 cruzado & 109 & 1.3 & $13.6 \pm 9.0$ \\
Fuera de pista & 42 & 0.5 & $5.3 \pm 2.8$ \\
Total & $\mathbf{8 5 8 1}$ & $\mathbf{1 0 0}$ & \\
\hline
\end{tabular}

predominio de los golpes interceptados (87.6\%), en un porcentaje muy inferior se observan los errores no forzados $(5.5 \%)$, puntos ganadores $(4.7 \%)$ y errores forzados (2.1\%). Se observan diferencias significativas entre todos los tipos de resultado del golpe $(p<0.01)$ excepto entre los errores no forzados y puntos ganadores $(p=1.000)$.

\section{Zonas de golpeo}

La tabla 5 muestra las frecuencias de golpeo en función de la zona de la pista en la que se encuentra el jugador en el momento de la acción. Prácticamente la mitad de los golpes se ejecutan desde la zona de fondo (49.1\%), seguido de la zona de red (26.4\%) y la zona de transición a la red (19.3\%), se observan diferencias significativas entre estas tres zonas $(\mathrm{p}<0.05)$. En un segundo plano encontramos los golpes ejecutados exactamente en las mismas zonas de la pista, pero por el jugador de la zona opuesta (cambiados de lado), en este caso no se observan diferencias entre las tres zonas ( $p$ > 0.05). Los golpes ejecutados por los jugadores desde fuera de la pista tienen una frecuencia de aparición muy escasa $(0.5 \%)$, mostrando diferencias significativas con el resto de las zonas de golpeo $(\mathrm{p}<0.01)$. 
Tabla 6. Tipo de golpe y su resultado.

\begin{tabular}{|c|c|c|c|c|c|c|c|c|c|c|c|c|c|}
\hline & \multirow[b]{3}{*}{ Tipo de Golpe } & \multicolumn{12}{|c|}{ Resultado del Golpe } \\
\hline & & \multicolumn{3}{|c|}{ PG } & \multicolumn{3}{|c|}{$\mathrm{EF}$} & \multicolumn{3}{|c|}{ GI } & \multicolumn{3}{|c|}{ ENF } \\
\hline & & $\mathrm{N}$ & $\%$ & $\mathrm{M} \pm \mathrm{DE}$ & $\mathrm{N}$ & $\%$ & $M \pm D E$ & $\mathrm{~N}$ & $\%$ & $M \pm D E$ & $\mathrm{~N}$ & $\%$ & $\mathrm{M} \pm \mathrm{DE}$ \\
\hline \multirow{5}{*}{$\begin{array}{l}\text { Golpes } \\
\text { Directos }\end{array}$} & Volea de revés & 48 & 3.4 & $6.0 \pm 1.1$ & 37 & 2.6 & $4.6 \pm 1.7$ & 1294 & 90.6 & $161.8 \pm 41.2$ & 49 & 3.4 & $6.1 \pm 2.9$ \\
\hline & Volea de derecha & 63 & 5.7 & $7.9 \pm 3.4$ & 24 & 2.2 & $3.0 \pm 1.7$ & 974 & 87.4 & $121.8 \pm 32.5$ & 54 & 4.8 & $6.8 \pm 3.2$ \\
\hline & Bandeja & 18 & 3.5 & $2.3 \pm 1.5$ & 1 & 0.2 & $0.1 \pm 0.3$ & 479 & 93.2 & $59.9 \pm 30.4$ & 16 & 3.1 & $2.0 \pm 1.3$ \\
\hline & Remate & 231 & 45.1 & $28.9 \pm 6.4$ & 4 & 0.8 & $0.5 \pm 0.7$ & 247 & 48.2 & $30.1 \pm 8.3$ & 30 & 5.9 & $3.8 \pm 2.2$ \\
\hline & Finta de remate & 7 & 9.2 & $0.9 \pm 1.3$ & 0 & 0 & 0 & 65 & 85.5 & $8.1 \pm 4.3$ & 4 & 5.3 & $0.5 \pm 0.7$ \\
\hline \multirow{4}{*}{$\begin{array}{l}\text { Golpes } \\
\text { Indirectos }\end{array}$} & Revés & 8 & 0.8 & $1.0 \pm 0.7$ & 16 & 1.5 & $2.0 \pm 1.3$ & 974 & 91.9 & $121.8 \pm 37.2$ & 62 & 5.8 & $7.8 \pm 3.2$ \\
\hline & Primer servicio & 1 & 0.1 & $0.1 \pm 0.3$ & 0 & 0 & 0 & 862 & 89.3 & $107.8 \pm 23.1$ & 102 & 10.6 & $12.8 \pm 3.6$ \\
\hline & Derecha & 8 & 0.9 & $1.0 \pm 1.0$ & 15 & 1.6 & $1.9 \pm 2.3$ & 849 & 92.9 & $106.1 \pm 23.7$ & 42 & 4.6 & $5.3 \pm 2.1$ \\
\hline & Segundo servicio & 0 & 0 & 0 & 0 & 0 & 0 & 95 & 97.9 & $11.9 \pm 2.6$ & 2 & 2.1 & $0.3 \pm 0.4$ \\
\hline \multirow{8}{*}{$\begin{array}{l}\text { Golpes } \\
\text { Indirectos con } \\
\text { Pared Simple }\end{array}$} & Salida de pared de derecha & 4 & 0.7 & $0.5 \pm 0.5$ & 18 & 3.4 & $2.3 \pm 1.9$ & 483 & 90.4 & $60.4 \pm 8.9$ & 29 & 5.4 & $3.6 \pm 2.6$ \\
\hline & Salida de pared de revés & 1 & 0.3 & $0.1 \pm 0.1$ & 8 & 2.3 & $1.0 \pm 0.9$ & 316 & 91.1 & $39.5 \pm 15.1$ & 22 & 6.3 & $2.8 \pm 1.9$ \\
\hline & Bajada de pared de derecha & 15 & 7.9 & $1.9 \pm 1.4$ & 0 & 0 & 0 & 158 & 83.6 & $19.8 \pm 5.8$ & 16 & 8.5 & $2.0 \pm 1.3$ \\
\hline & Pared lateral de revés & 1 & 0.6 & $0.1 \pm 0.3$ & 2 & 1.3 & $0.3 \pm 0.4$ & 139 & 89.7 & $17.4 \pm 8.3$ & 13 & 8.4 & $1.6 \pm 1.6$ \\
\hline & Reja de revés & 1 & 1.2 & $0.1 \pm 0.3$ & 12 & 14 & $1.5 \pm 1.8$ & 71 & 82.6 & $8.9 \pm 3.7$ & 2 & 2.3 & $0.3 \pm 0.4$ \\
\hline & Pared lateral de derecha & 0 & 0 & 0 & 0 & 0 & 0 & 69 & 93.2 & $8.6 \pm 7.7$ & 5 & 6.8 & $0.6 \pm 0.9$ \\
\hline & Bajada de pared de revés & 0 & 0 & 0 & 0 & 0 & 0 & 32 & 97 & $4.0 \pm 1.4$ & 1 & 3 & $0.1 \pm 0.3$ \\
\hline & Reja de derecha & 0 & 0 & 0 & 0 & 0 & 0 & 23 & 95.8 & $3.3 \pm 1.9$ & 1 & 4.2 & $0.1 \pm 0.4$ \\
\hline \multirow{8}{*}{$\begin{array}{l}\text { Golpes } \\
\text { Indirectos con } \\
\text { Doble Pared }\end{array}$} & Doble pared que abre con giro de derecha & 1 & 1.2 & $0.1 \pm 0.3$ & 10 & 12 & $1.3 \pm 1.3$ & 67 & 80.7 & $8.4 \pm 5.6$ & 5 & 6 & $0.6 \pm 0.7$ \\
\hline & Doble pared que cierra de revés & 0 & 0 & 0 & 2 & 2.7 & $0.3 \pm 0.4$ & 68 & 90.7 & $8.5 \pm 3.1$ & 5 & 6.7 & $0.6 \pm 0.7$ \\
\hline & Doble pared que abre de revés & 0 & 0 & 0 & 3 & 5.4 & $0.4 \pm 0.5$ & 53 & 94.6 & $6.6 \pm 2.2$ & 0 & 0 & 0 \\
\hline & Doble pared que abre de derecha & 0 & 0 & 0 & 2 & 5.7 & $0.3 \pm 0.4$ & 28 & 80 & $3.5 \pm 2.1$ & 5 & 14.3 & $0.6 \pm 0.9$ \\
\hline & Doble pared que cierra de derecha & 0 & 0 & 0 & 0 & 0 & 0 & 21 & 91.3 & $2.6 \pm 2.9$ & 2 & 8.7 & $0.3 \pm 0.4$ \\
\hline & Doble pared que abre con giro de revés & 0 & 0 & 0 & 4 & 26.7 & $0.5 \pm 0.7$ & 11 & 73.3 & $1.4 \pm 1.6$ & 0 & 0 & 0 \\
\hline & Doble pared que cierra con giro de revés & 0 & 0 & 0 & 0 & 0 & 0 & 15 & 100 & $1.9 \pm 1.8$ & 0 & 0 & 0 \\
\hline & Doble pared que cierra con giro de derecha & 0 & 0 & 0 & 0 & 0 & 0 & 1 & 50 & $0.1 \pm 0.3$ & 1 & 50 & $0.1 \pm 0.3$ \\
\hline \multirow{5}{*}{$\begin{array}{l}\text { Golpes } \\
\text { Indirectos } \\
\text { Contra-Pared }\end{array}$} & Contra-pared fondo de derecha & 0 & 0 & 0 & 14 & 10.9 & $1.8 \pm 1.3$ & 109 & 84.5 & $13.6 \pm 5.5$ & 6 & 4.7 & $0.8 \pm 0.8$ \\
\hline & Contra-pared fondo de revés & 0 & 0 & 0 & 4 & 30.8 & $0.5 \pm 0.7$ & 8 & 61.5 & $1.0 \pm 1.4$ & 1 & 7.7 & $0.1 \pm 0.3$ \\
\hline & Contra pared lateral de revés & 0 & 0 & 0 & 0 & 0 & 0 & 7 & 87.5 & $0.9 \pm 0.9$ & 1 & 12.5 & $0.1 \pm 0.3$ \\
\hline & Contra-pared lateral de derecha & 0 & 0 & 0 & 1 & 25 & $0.1 \pm 0.3$ & 3 & 75 & $0.4 \pm 0.5$ & 0 & 0 & 0 \\
\hline & Total & 407 & 4.7 & & 177 & 2.1 & & 7521 & 87.6 & & 476 & 5.5 & \\
\hline
\end{tabular}

$\mathrm{PG}=$ Punto ganador; $\mathrm{EF}=$ Error forzado; $\mathrm{Gl}=$ Golpe interceptado; $\mathrm{ENF}=$ Error no forzado.

Relación entre los tipos de golpe y su consecuencia

La tabla 6 muestra los tipos de golpe y su resultado. Los porcentajes más elevados de puntos ganadores se registraron con el remate (45.1\%), en menor medida encontramos la finta de remate (9.2\%), la bajada de pared de derecha (7.9\%), la volea de derecha (5.7\%), la bandeja (3.5\%) y la volea de revés (3.4\%). En relación a los errores no forzados encontramos un alto índice en la doble pared que cierra con giro de derecha (50\%), doble pared de derecha (14.3\%), contra pared lateral de revés (12.5\%), doble pared que cierra de derecha y pared lateral de revés (8.4\%). Cabe destacar que en su mayoría son golpes que no presentan una frecuencia de aparición mayor al 1\%. En cuanto a errores forzados destacan el golpe de contra-pared de fondo de revés $(30.8 \%)$, doble pared que abre con giro de revés (26.7\%), contra-pared lateral de derecha (25\%), reja de revés (14\%) y doble pared que abre con giro de derecha (12\%). Al igual que en el caso anterior, varios de estos golpeos se suceden con una frecuencia inferior al $1 \%$.

En puntos ganadores existen diferencias significativas entre todos los golpes directos excepto volea de derecha y revés $(\mathrm{p}=0.279)$. Con golpes indirectos no 
existen diferencias entre derecha y revés y primer servicio ( $p>0.05)$. En paredes simples encontramos diferencias entre todos los golpes $(p<0.05)$ excepto entre salida pared revés, pared lateral revés y reja de revés ( $p$ > 0.05). En cuanto a errores no forzados, no se han hallado diferencias entre los golpes directos de volea de derecha y volea de revés $(\mathrm{p}=0.105)$ ni entre bandeja y remate $(p=0.382)$. Sin embargo, existen diferencias entre todos los golpes indirectos $(p<0.05)$ excepto entre revés y primer servicio ( $p>0.05)$. En paredes simples, no hay diferencias significativas entre salida de pared de derecha, de revés, bajada de pared de derecha, pared lateral de derecha y pared lateral de revés, ni entre reja de revés, pared lateral de derecha, pared lateral de revés y reja de derecha ni entre pared lateral de revés y pared lateral de derecha ( $p$ > 0.05). En dobles paredes y contra-paredes no se han hallado diferencias significativas entre golpes ( $p>0.05)$. En errores forzados, no existen diferencias entre golpes directos volea de derecha y volea de revés $(\mathrm{p}=0.105)$ ni entre bandeja y remate $(p=0.382)$. Tampoco se han hallado diferencias entre golpes indirectos de derecha y revés ( $p=0.505)$. En golpes con pared simple únicamente existen diferencias significativas entre la salida de pared de recha y la pared lateral de revés $(\mathrm{p}=0.010)$. En los golpeos con doble pared no hay diferencias significativas entre ninguno de los golpes ( $\mathrm{p}>0.05$ ). Asimismo, en los golpeos de contra-pared tampoco se han hallado diferencias entre contra-pared de fondo de derecha y revés, ni entre contra-pared de fondo de revés y contra-pared lateral de derecha ( $p>0.05)$. En cuanto a golpes interceptados, existen diferencias entre todos los golpes directos $(p<0.05)$ excepto entre bandeja y remate $(p=0.192)$. En los golpes indirectos, no existen diferencias significativas entre revés, derecha y primer servicio ( $p>0.05$ ), mientras que sí se han encontrado entre estos tres golpes y el segundo servicio ( $p$ < 0.05). En paredes simples, no existen diferencias entre salida de pared de derecha y revés ( $p$ $=0.171$ ) ni entre salida de pared de revés y bajada de pared de derecha $(p=0.629)$, ni entre bajada de pared de derecha pared lateral de derecha y revés ( $p$ > 0.05), ni entre bajada de pared de revés, pared lateral de derecha, pared lateral de revés, reja de derecha y reja de revés ( $p$ > 0.05). En paredes dobles, no se han hallado diferencias significativas entre doble pared que abre con giro de derecha, doble que abre con giro de revés y doble pared que abre de revés, ni entre doble pared que abre de recha, doble pared que cierra de derecha y doble pared que cierra con giro de revés ( $p>0.05$ ), ni entre doble pared que abre con giro de revés y doble pared que cierra con giro de derecha ( $p=0.065)$. En los golpes de contra-pared, no existen diferencias entre contra-pared de fondo de derecha, contra-pared lateral de revés y contra-pared lateral de derecha, sin embargo, se ha hallado diferencias significativas entre la contra-pared de fondo de derecha y el resto de los golpes de contra-pared $(\mathrm{p}<0.05)$.

\section{Discusión}

Este es el primer estudio que aporta información detallada sobre las acciones de juego en pádel masculino profesional basándose en el análisis de las características de cada golpe, tales como la tipología de los golpes, zonas desde la que se realizan, trayectoria de la bola, profundidad y el resultado de cada golpeo. Asimismo, se analizan las diferencias entre los diferentes tipos de golpeo y las variables observadas.

En cuanto a la tipología de los golpes, cabe destacar la importancia de las voleas las cuales muestran una presencia significativamente superior al resto de golpes y representaron un $29.6 \%$ del total (16.6\% volea de revés y $13.0 \%$ volea de derecha) que se ejecutan en un partido de pádel, cifra muy próxima al $29.8 \%$ obtenido por Torres-Luque et al. (2015) y al $29.4 \%$ obtenido por Almonacid (2012). Priego et al. (2013) y Sañudo et al. (2008) obtuvieron porcentajes algo inferiores, $24.6 \%$ y $25.5 \%$ respectivamente. El objetivo principal de los jugadores en pádel es alcanzar la red para conseguir los puntos (Courel, Sánchez-Alcaraz \& Cañas, 2015), ya que el $80 \%$ de ellos se anotan desde esta posición, lo cual justificaría el elevado número de golpeos directos. Asimismo, el predominio de las voleas también podría atribuirse a la combinación de dos factores, por un lado, a la distribución del número de golpes que se ejecutan desde cada zona de la de la pista y, por otro lado, a la tipología de golpe que es posible realizar en cada una de ellas. Así, el número de golpes se distribuye exactamente entre el $50 \%$ en fondo (defensa) y el otro 50\% entre red-transición (Almonacid, 2012). La tipología de los golpes que es posible ejecutar en las zonas de red (voleas, remate y bandeja) y transición (voleas, remate, bandeja, derecha, revés y alambradas), es muy inferior al repertorio de golpes defensivos existentes (derecha, revés, salidas de pared, bajadas de pared, dobles paredes, paredes laterales, etc.), lo cual produce un aumento significativo en el número de voleas.

En cuanto a los golpes de fondo y transición, poseen un gran peso estadístico los golpes de derecha y revés $10.7 \%$ y $12.4 \%$ respectivamente, para los que Almonacid (2012) registró porcentajes del 11.2\% y 13.1\%. Con el fin de poder contrastar datos con las publicaciones de Sañudo et al. (2008) y Priego et al. (2013), se sumó 
el total de los diferentes golpes de derecha (directos e indirectos). Para todos los tipos de derecha, se obtuvo un porcentaje del $23.5 \%$ sobre la totalidad de los golpeos, cifra superior al $16.4 \%$ obtenido por Priego et al. (2013) e inferior al 31.9\% de Sañudo et al. (2008). Lo mismo ocurre en los golpeos de revés, en nuestro caso representaron el $21.9 \%$ del total de los golpeos, frente a un $17.3 \%$ en el caso de Priego et al. (2013) y un $23.9 \%$ en Sañudo et al. (2008). También sobresalen estadísticamente los golpes de remate y bandeja, que se producen con una frecuencia del $12 \%$ (6\% cada uno). En los estudios de Priego et al. (2013) y Sañudo et al. (2008) se obviaron las bandejas, registrando únicamente remates, con porcentajes del $20.6 \%$ y $13.8 \%$ respectivamente. Presuponemos que se sumaron con los golpes de bandeja dadas las elevadas cifras en el total de remates. Estas variaciones en los porcentajes, consideramos que pueden ser debidas a las diferencias entre los instrumentos observacionales que se han diseñado para cada estudio, ya que las nomenclaturas y sistemas de clasificación no son homogéneas, lo cual nos plantea la necesidad de estandarizar una herramienta que permita homogeneizar el proceso de observación y análisis técnico-táctico.

En cuanto a las trayectorias de los golpes, los datos evidencian que existe un predominio del juego cruzado (57.2\%) sobre el paralelo (42.3\%), existiendo diferencias significativas entre ambos tipos de trayectoria. Sin embargo, consideramos que estas cifras no se deben a aspectos tácticos del juego, sino al reglamento del propio deporte. La normativa establece que los saques deben seguir una trayectoria cruzada para ser válidos, es por ello que la frecuencia de las trayectorias paralelas aumenta del $42.3 \%$ al $48.1 \%$ y las cruzadas disminuyen del $57.2 \%$ al $51.3 \%$ cuando prescindimos de los saques. Por tanto, se deduce que el flujo direccional del juego tiende a ser homogéneo una vez que la bola ha sido puesta en juego.

En referencia al resultado de los golpeos, se obtuvieron porcentajes del $87.6 \%$ de golpes interceptados, $5.5 \%$ de errores no forzados, $4.7 \%$ de puntos ganadores y $2.1 \%$ de errores forzados. El elevado porcentaje de golpes interceptados pone de manifiesto que el pádel es un deporte de intercambios prolongados, con duraciones medias por punto de 11.6 segundos (Sánchez-Alcaraz, 2014). Asimismo, destaca el hecho de que no se observan diferencias entre el porcentaje de errores forzados y puntos ganadores. Estos resultados resaltan la importancia de regular el umbral de riesgo asumido. No obstante, será el perfil del jugador el que determine la cantidad de riesgo que puede asumir, que variará en función de la relación entre los puntos ganadores y errores no forzados que comete.
Centrándonos en la finalización de los puntos, cabe destacar la importancia de los errores no forzados, que representan la mayoría del final de los puntos (44.9\%), seguido de los puntos ganadores (38.4\%) y por último los errores forzados (16.7\%). Estas cifras difieren de las obtenidas por Couriel, Sánchez-Alcarzaz \& cañas (2015), en el que se invierten los dos primeros parámetros, pasando a ser los puntos ganadores la principal forma de finalización de los puntos $(44.3 \%$ de puntos ganadores, $32.24 \%$ de errores no forzados y $23.45 \%$ errores forzados). Estas divergencias pueden deberse al registro de los errores no forzados, que a presar de ser un término definido y profundamente estudiado en la bibliografía científica de otros deportes de raqueta (Krames, 2009), en ocasiones puede variar a juicio del observador. Sin embargo, los datos que se han registrado en la presente investigación se corresponden con lo estudiado hasta la fecha en otros deportes de raqueta como el tenis, en el que se mantiene este orden, y en el cual se ha observado que, a mayor nivel de rendimiento, más tienden a igualarse el número de puntos ganadores al de errores no forzados (Brody, 2006).

Por otra parte, la estadística de las zonas desde las que los jugadores golpean a la bola arrojó datos de frecuencia del $49.1 \%$ de golpeos desde el fondo de la pista, $26.4 \%$ desde la red y $19.3 \%$ desde la zona intermedia o de transición, existiendo diferencias significativas en el número de golpeos efectuados desde cada zona ( $\mathrm{p}<0.05)$. Hasta la fecha, estos parámetros sólo han sido estudiados por Almonacid (2012), el cual segmentó las zonas de la pista en red, pared y fondo, obteniendo valores del $50 \%$ en red y $50 \%$ en defensa (paredes y fondo), lo cual se ajusta a los datos que hemos registrado en este estudio. Courel, Sánchez-Alcaraz \& Cañas (2015) también analizaron zonas de golpeo, sin embargo, en su caso registraron solo aquellos golpes con los que se finaliza el punto, por lo que no podemos realizar un análisis comparativo de los resultados.

La prevalencia de los golpeos de fondo sobre zona intermedia y red se repite en las bolas en las que los jugadores golpean desde el lado natural del compañero. Sumando los porcentajes por zona, obtenemos frecuencias del $50.9 \%$ para la zona de fondo, $27.7 \%$ para la red y $21 \%$ para la zona de transición. Al aislar las bolas golpeadas por los jugadores en el lado de la pista ocupado por el compañero (zonas cambiadas), se observa que la zona de red pasa de ser la segunda zona desde la que más se golpea a la tercera (1.3\%) y la zona de transición adquiere una mayor importancia (1.7\%), llegando prácticamente a igualarse con el fondo de pista (1.8\%). Estos resultados sugieren que, en ciertas situaciones los jugadores adquieren roles diferenciados, 
cubriendo uno de ellos la zona de fondo al completo y no únicamente su lado natural de la pista, con el fin de que el compañero pueda permanecer en zona de ataque y así evitar la pérdida de la red.

Una vez más, los datos expuestos para la realidad del juego se modifican sustancialmente al excluir el primer y segundo servicio del análisis, pasando a ser $42 \%$ de golpeos desde el fondo de pista, $30.1 \%$ para la red y 21.9\% para la zona de transición. En último lugar encontramos las bolas golpeadas desde fuera de la pista, que suponen el $0.5 \%$ del total de los golpeos.

Por otro lado, se consideró fundamental llevar a cabo un análisis de los resultados de los golpeos en función de su tipología. Podemos afirmar que el golpe más efectivo en pádel para ganar el punto es el remate, con un porcentaje de $45.1 \%$ de puntos ganadores a costa de un ratio relativamente bajo de errores no forzados (5.9\%). Esto es debido a que se trata de un golpe muy agresivo y seguro cuando se domina la técnica (Llamas, García \& Pérez, 2013), hecho que se da por supuesto en el pádel profesional. Los jugadores aprovechan la potencia de este golpeo, con trayectoria descendiente, para sacar la bola de la pista por el fondo (4 metros) en el $6.3 \%$ de los remates, siendo punto ganador el $100 \%$ de los casos dada la imposibilidad por parte del rival de alcanzar la bola una vez que ha salido. Asimismo, se han encontrado diferencias significativas entre todos los tipos de golpes de red, exceptuando entre las voleas de derecha y revés, lo cual pone de manifiesto que ambas ofrecen la misma efectividad para ganar el punto.

Entre los tipos de golpe más efectivos también destaca la bajada de pared de derecha, que a pesar de ser un golpe ejecutado desde el fondo de pista finaliza en un $7.9 \%$ de las ocasiones en punto ganador, sin embargo, el riesgo tomado por el jugador en este caso es bastante mayor que en el remate, reflejándose en un $8.5 \%$ de errores no forzados. En un tercer plano de efectivi- dad encontramos las voleas de derecha y revés $(5.7 \%$ y $3.4 \%$ de puntos ganadores respectivamente) y las bandejas (3.5\% de puntos ganadores). Sin embargo, son golpes bastante seguros ya que presentan un ratio de errores no forzados del $4.8 \%$ en la volea de derecha, $3.4 \%$ en la de revés y $3.1 \%$ en el caso de la bandeja.

\section{Conclusiones}

Los golpes más frecuentes en pádel son las voleas, derechas, reveses, bandejas y en última instancia remates. Existe un predominio significativo del juego cruzado sobre el paralelo, en gran parte determinado por la obligatoriedad direccional del saque. El elevado porcentaje de golpes interceptados pone de manifiesto que los puntos implican largos intercambios, que finalizan con errores no forzados y golpes ganadores en igual proporción. A pesar de ser un deporte en el que los puntos se ganan por lo general en la red, existe un predominio significativo de los golpes efectuados desde el fondo de pista, sobre la zona de transición y red. Los golpes más efectivos para ganar los puntos son el remate, las voleas, la bandeja y las bajadas de pared.

El presente estudio se ha centrado en el desarrollo de una herramienta computerizada de análisis técnico-táctico en pádel, proponiéndose como objetivo secundario el análisis global del juego a nivel profesional. No obstante, no se analizan las relaciones entre las diferentes variables registradas, como, por ejemplo, la trayectoria del golpe y la eficacia de este o el recorrido de la bola y la forma de finalización del punto. Por lo tanto, se proponen como futuras líneas de investigación el análisis de las relaciones de dependencia entre variables, así como la contextualización de estas según la situación del partido: tanteo, marcador o tiempo de juego. 


\section{BI B LIOG RAFÍA}

Almonacid, B. (2012). Perfil de juego en pádel de alto nivel. (Tesis doctoral inédita). Universidad de Jaén, Jaén.

Courel, J., Cañas, J.C., \& Sánchez-Alcaraz, B.J. (2016). Valoración de la precisión del golpeo en jugadores de pádel en función de su nivel de juego. RICYDE: Revista Internacional de Ciencias del Deporte, 45(13), 324-333. doi:10.5232/ricyde2016.04507

Courel, J., Sánchez-Alcaraz, B.J., \& Cañas, J. (2015). Effectiveness at the net as a predictor of the final match outcome in professional padel players. International Journal of Performance Analysis in Sport, 15, 632640.

Brody, H. (2006). Unforced errors and error reduction in tennis. British Journal of Sport Medicine, 40(5), 324-333. doi:10.1136/ bjsm.2005.023432

Cabello, D., \& Gonzalez, J. J. (2003) Analysis of the characteristics of competitive badminton. British Journal of Sports Medicine, 37(1), 6266. doi:10.1136/bjsm.37.1.62

Carrasco, L., Romero, S., Sañudo, B., \& De Hoyo, M. (2011). Game analysis and energy requirements of paddle tennis competition. Science and Sports, 26, 338-344. doi:10.1016/j.scispo.2010.12.016

Cohen, J. (1968). Weighted kappa: Nominal scale agreement provision for scaled disagreement or partial credit. Psychological Bulletin, 70(4), 213. doi: $10.1037 / \mathrm{h} 0026256$

Costa, T.J., Yuzo, F., Tardelli, M., Roveri, C.L., Shoiti, M., Machado, R., \& Arruda, F. (2016). Analysis of the distances covered and technical actions performed by professional tennis players during official matches. Journal of Sports Sciences, 35(4), 361-364. doi:10.1080/026 40414.2016.1165858.

Courel-Ibáñez, J., Sánchez-Alcaraz, B.J., García, S., \& Echegaray, M. (2017). Evolución del pádel en España en función del género y edad de los practicantes. Cultura, Ciencia y Deporte, 34(12) 39-46. doi:10.12800/ccd.v12i34.830

Courel-Ibáñez, J. \& Sánchez-Alcaraz, B.J. (2017). Efecto de las variables situacionales sobre la duración y eficacia de los puntos en jugadores de pádel de élite. Apunts, Educación Física y Deportes, 127, 72-78. doi:10.5672/apunts.2014-0983.es.(2017/1).127.07

Courel, J., Cañas, J.C., Sánchez-Alcaraz, B.J., \& Alarcón-Guerrero, R. (2014). Investigación en pádel (Volumen I). Murcia: Universidad de Murcia, Servicio de Publicaciones.

Federación Española de Pádel (noviembre, 2015). Evolución de las licencias jugador/a últimos años. Recuperado de http://www.padelfederacion.es/Datos_Federacion.asp? $\mathrm{Id}=0$

Fernández, J., Méndez-Villanueva, A., \& Pluim, B.M. (2006). Intensity of tennis match play. British Journal of Sports Medicine, 40(1), 387-391. doi:10.1136/bjsm.2005.023168

Fernández de Ossó, A.I., \& León-Prados, J.A. (En prensa). Herramienta de evaluación técnico-táctica en pádel. Revista Internacional de Medicina y Ciencias de la Actividad Física y del Deporte. doi:10.15366/ rimcafd2017.68.008

Gabín, B., Camerino, O., Anguera, M. T., \& Castañer, M. (2012). Lince: multiplatform sport analysis software. Procedia-Social and Behavioral Sciences, 46, 4692-4694. doi:10.1016/j.sbspro.2012.06.320

García-Benítez, S., Pérez-Bilbao, T., Echegaray, M., \& Felipe, J.L. (2016). Influencia del género en la estructura temporal y las acciones de juego del pádel profesional. Cultura, Ciencia y Deporte, 33(11), 241-247. doi:10.12800/ccd.v11i33.769

Girard, O., Chevalier, R., Habard, M., Sciberras, P., Hot, P., \& Millet, G.P. (2007). Game analysis and energy requirements of elite squash. Journal of strength and Conditioning Research, 21(3), 909-914. doi:10.1519/R-20306.1

González-Carvajal, C. (2003). Conocer el deporte. Pádel. Madrid: Tutor.

Gorospe, G., Hernández, A., Anguera, M. T., \& Martínez, R. (2005). Desarrollo y optimización de una herramienta observacional en el tenis de individuales. Psicothema, 17(1), 123-127.
Hong, Y., \& Tong, Y. M. (2000). The playing pattern of the world's top single badminton players in competition - a notation analysis. Journal of Human Movement Studies, 38, 195-200.

Hughes, M., \& Clarke, S. (1995). Surface effect on elite tennis strategy. En Science and Racket Sports (editado por T. Reilly, M. Hughes and A. Lees), pp. 272-277. London: E \& FN Spon.

Johnson, C., \& McHugh, M. (2006). Performance demands of professional male tennis players. British Journal of Sports Medicine, 40(8), 696-699. doi:10.1136/bjsm.2005.021253

Kilit, B., Şenel, Ö., Arslan, E., \& Can, S. (2016). Physiological responses and match characteristics in professional tennis players during a onehour simulated tennis match. Journal of Human Kinetics, 51, 83-92. doi:10.1515/hukin-2015-0173

Krames, J. (2009). The unforced error. Nueva York: Penguin books.

Llamas, V., García, E., \& Pérez, J. (2013). Nivel de ejecución del remate de potencia de pádel en alumnos de la Universidad de Murcia. Esmáf, revista digital de educación física, 23(4), 16-24.

Moncaut, A. P. (2003). Fundamentos Técnicos del Pádel. Madrid: Gymnos. Moyano, J. (2011). 1001 Juegos y ejercicios de pádel. Sevilla: Wancuelen.

Muñoz-Marín, D., Courel-Ibáñez, J., Sánchez-Alcaraz, B.J., Díaz, J., Grijota F.J., \& Muñoz, J. (2017). Análisis del uso y eficacia del globo para recuperar la red en función del contexto de juego en pádel. Retos, Nuevas tendencias en Educación Física, Deporte y Recreación, 31, 19-22.

Navarro, S. (2014). Fundamentos del pádel. Los secretos de un entrenamiento eficaz para deportistas. Barcelona: Paidotribo.

O’Donoghe, P., \& Ingram, B. (2001). A notational analysis of elite tennis strategy. Journal of Sport Science, 19(2), 107-115. doi:10.1080/026404101300036299

Over, S., \& O'Donoghue, P. (2008). Whats the point - Tennis analysis and why. ITF Coaching and Sport Science Review, 15(45), 19-21.

Priego, J. I., Olaso Melis, J., Llana-Belloch, S., Pérez-Soriano, P., González García, J. C., \& Sanchís Almenara, M. (2013). Padel: A Quantitative study of the shots and movements in the high-performance. Journal of Human Sport and Exercise, 8(4), 925-931. doi:10.4100/ jhse.2013.84.04

Ramón-Llin, J. S., Guzmán, J. F., Llana Belloch, S., Vučković, G., \& James, N. (2013). Comparison of distance covered in paddle in the serve team according to performance level. Journal of Human Sport and Exercise, 8(3), 738-742. doi:10.4100/jhse.2013.8.Proc3.20

Reid, M., \& Duffield, R. (2014). The development of fatigue during match-play tennis. British Journal of Sports Medicine, 48(1), i7-i11. doi:10.1136/bjsports-2013-093196.

Ruiz Barquín, R., \& Lorenzo García, Ó. (2008). Características psicológicas en los jugadores de pádel de alto rendimiento. Revista iberoamericana de psicología del ejercicio y el deporte, 3(2), 183-200.

Sánchez-Alcaraz, B.J. (2013). Historia del pádel. Materiales para la historia del deporte, 11, 57-60.

Sánchez-Alcaraz, B.J. (2014a). Análisis de la exigencia competitiva del pádel en jóvenes jugadores. Kronos, 13(1), 1-7.

Sánchez-Alcaraz, B. J. (2014b). Diferencias en las acciones de juego y la estructura temporal entre el pádel masculino y femenino profesional. Acción motriz, 12, 17-22.

Sanchis, J., González, J. C., López, J. A., Dorado, C., \& Chavarren, J. (1998). Propuesta de un modelo de entrenamiento de squash a partir de parámetros obtenidos durante la competición. Apunts Educació Física i Esports, 52, 43-52.

Sañudo, B., De Hoyo-Lora, M., \& Carrasco-Páez, L. (2008). Demandas fisiológicas y características estructurales de la competición en pádel masculino. Apunts Educació Física i Esports, 94(4), 23-28. 